\title{
Modelling cathode spots in glow discharges in the cathode boundary layer geometry
}

\author{
P. G. C. Almeida, M. S. Benilov, and M. S. Bieniek \\ Departamento de Física, Universidade da Madeira, \\ Largo do Município, 9000 Funchal, Portugal, and \\ Instituto de Plasmas and Fusão Nuclear, IST, \\ Universidade de Lisboa, Portugal
}

\begin{abstract}
Self-organized patterns of cathode spots in glow discharges are computed in the cathode boundary layer geometry, which is the one employed in most of the experiments reported in the literature. The model comprises conservation and transport equations of electrons and a single ion species, written in the drift-diffusion and local-field approximations, and Poisson's equation. Multiple solutions existing for the same value of the discharge current and describing modes with different configurations of cathode spots are computed by means of a stationary solver. The computed solutions are compared to their counterparts for plane-parallel electrodes, and experiments. All of the computed spot patterns have been observed in the experiment.
\end{abstract}

\section{Introduction}

Self-organized patterns of cathode spots in DC glow microdischarges were observed for the first time in 2004 [1] and represent an important and interesting phenomenon. A range of experimental reports have since been published [1-10]. Modelling of the phenomenon [9, 11-15] has revealed, in agreement with the general theory of cathode spots and patterns in arc and DC glow discharges [16], the existence of multiple steady-state solutions for a given value of discharge current, which comprise modes of current transfer associated with different cathode spot patterns. Predictions concerning the existence of self-organizaed patterns in gases other than xenon, generated from the modelling, have been confirmed experimentally [9].

The vast majority of the experiments [1-10] have been performed in a discharge device comprising a flat cathode and a ring-shaped anode, separated by a dielectric (cf., e.g., figure 1 of [10]); this discharge configuration is called cathode boundary layer discharge (CBLD) by the authors of the experiment. However, the modelling has been performed up to date for discharges with parallel-plane electrodes only [9, 11-15]. The question of how the shape of the anode affects the pattern of self-organization has so far not been addressed. Furthermore, the effect over 3D spots of absorption of the charged particles 
by a dielectric surface has not been investigated in full due to computational difficulties $[14]$.

In this work, 3D modelling of cathode spots is reported for the first time in CBLD, and the full pattern of self-organization is computed with account of absorption of charged particles at the dielectric surface. The outline of the paper is as follows. The model is described in section 2. In section 3.1 the effect over the fundamental mode of the discharge radius, the thickness of the cathode and dielectric, and of a dielectric surface that reflects charged particles is investigated. In section 3.2 examples of computed 3D modes are given and compared to their experimental counterparts. In section 3.3 the effect on 3D modes of a dielectric surface that reflects charged species is investigated. In section 4 conclusions are drawn.

\section{$2 \quad$ Model and Numerics}

The model employed in this work is the most basic self-consistent model of glow discharge. Although the model is very well-known, it is described here for the sake of completeness. The model comprises equations for conservation of electrons and a single ion species, written in the drift-diffusion transport approximation, and Poisson's equation.

$$
\begin{aligned}
\nabla \cdot \mathbf{J}_{i} & =n_{e} \alpha \mu_{e} E-\beta n_{e} n_{i}, \quad \mathbf{J}_{i}=-D_{i} \nabla n_{i}-n_{i} \mu_{i} \nabla \varphi, \\
\nabla \cdot \mathbf{J}_{e} & =n_{e} \alpha \mu_{e} E-\beta n_{e} n_{i}, \quad \mathbf{J}_{e}=-D_{e} \nabla n_{e}+n_{e} \mu_{e} \nabla \varphi, \\
\varepsilon_{0} \nabla^{2} \varphi & =-e\left(n_{i}-n_{e}\right) .
\end{aligned}
$$

Here $n_{i}, n_{e}, \mathbf{J}_{i}, \mathbf{J}_{e}, D_{i}, D_{e}, \mu_{i}$, and $\mu_{e}$ are number densities, densities of transport fluxes, diffusion coefficients, and mobilities of the ions and electrons, respectively; $\alpha$ is Townsend's ionization coefficient; $\beta$ is coefficient of dissociative recombination; $\varphi$ is electrostatic potential, $E=|\nabla \varphi|$ is electric field strength; $\varepsilon_{0}$ is permittivity of free space; and $e$ is elementary charge. The local-field approximation is employed (i.e. electron transport and kinetic coefficients are assumed to depend on the local electric field only).

Boundary conditions at the cathode and anode are written in the conventional form. Diffusion fluxes of the attracted particles are neglected as compared to drift; the normal flux of the electrons emitted by the cathode is related to the flux of incident ions in terms of the effective secondary emission coefficient $\gamma$, which is assumed to characterize all mechanisms of electron emission (due to ion, photon, and excited atom bombardment) [17]; density of ions vanishes at the anode; electrostatic potentials of both electrodes are given. The dielectric surface is electrically insulating, and absorbs the charged particles (i.e. case $i$ ) $n_{i}=n_{e}=0$ ); for comparison, some solutions were computed for the case of a reflecting dielectric surface (i.e. case $i i) \mathbf{n} \cdot \nabla n_{i}=\mathbf{n} \cdot \nabla n_{e}=0$ ). With the computational domain from figure 1 , the boundary conditions read

$$
\begin{array}{rll}
\text { cathode }(A B): & \frac{\partial n_{i}}{\partial z}=0, \quad J_{e z}=-\gamma J_{i z}, \quad \varphi=0 ; \\
\text { anode }(C D E): & n_{i}=0, \frac{\partial n_{e}}{\partial n}=0, \quad \varphi=U ; \\
\text { dielectric }(B C): & \begin{array}{l}
\text { i) } n_{i}=n_{e}=0 \\
\text { ii) } \frac{\partial n_{i}}{\partial r}=\frac{\partial n_{e}}{\partial r}=0
\end{array}, J_{e r}-J_{i r}=0 .
\end{array}
$$


Here $U$ is the discharge voltage, the subscripts $r$ and $z$ denote radial and axial projections of corresponding vectors, and $\partial n_{e} / \partial n$ is the normal derivative of electron density. The lengths $D E$ and $A G$ are large enough so that boundaries $E F$ and $F G$ do not affect the solution. The control parameter can be either discharge voltage $U$ or discharge current $I$, depending on the slope of the current voltage-characteristics (CVC) $U(I)$.

Results reported in this work refer to a discharge in xenon under the pressure of 30 Torr. The (only) ionic species considered is $\mathrm{Xe}_{2}^{+}$. The transport and kinetic coefficients are the same as in [14]. A more detailed model (one that also took into account both atomic and molecular ions, excited atoms, excimers, stepwise ionization, ionization of excimers and non-locality of electron energy) resulted in qualitatively similar self-organized patterns [14], therefore the relatively simple model described in this section was seen as sufficient for the purposes of this investigation.

The problem (1) to (2) admits multiple solutions describing different discharge modes. One such mode exists for all ranges of current, it is 2D (axially symmetric) and termed fundamental, this is routine to calculate. 3D modes bifurcate from (and rejoin) the fundamental mode and are termed non-fundamental modes.

To calculate non-fundamental modes, one first locates points of bifurcation on the fundamental mode by means of linear stability analysis, the procedure is discussed in detail in [11]. Next a 3D calculation domain is created by rotating the 2D axially symmetric domain from figure 1 about the axis of symmetry, by an amount corresponding to the expected azimuthal periodicity of the 3D mode being sought. The beginning of the nonfundamental mode is then searched for on the fundamental mode, with the 3D calculation domain, in the vicinity of the bifurcation point predicted by the linear stability analysis. Small perturbations are introduced at the bifurcation point; the iterations will eventually converge to the 3D mode. The remainder of the 3D mode is straight-forward to calculate.

The above procedure was realized using stationary and eigenvalue solvers from the commercial product COMSOL Multiphysics. The time taken by the stationary solver to find convergence to one of the most computationally intensive 3D solutions is around 1 hour, with a computer with a Intel Core i7-4770 CPU at $3.4 \mathrm{GHz}$ and $32 \mathrm{~GB}$ of RAM.

\section{Results}

\subsection{Fundamental mode}

In figure 2, the CVC of the fundamental mode is displayed in four sets of conditions, labeled 1 to 4 in the figure. Surprisingly, two turning points and a loop are present on the CVC corresponfing to the baseline conditions, line 1.

The whole current range in figure 2 can be divided into three regions, marked I, II, III. At the top of the figure there is an illustration of the characteristic distribution of current density on the cathode surface for each region. The color range shown in the bar is used also for the rest of the document. The general pattern of evolution of the fundamental mode with increasing current is as follows. In region I, corresponding to the Townsend discharge, the current is distributed on the cathode in the form of a ring. In region II, the ring of current grows thicker with increasing current. In region III, corresponding to the abnormal discharge, the discharge fills most of the cathode surface.

In the case represented by line 1 , a pattern with a central spot appears on the section 
between the turning points, as indicated in the figure. This transition is accompanied by a loop in the CVC. The loop is absent in the CVC of cases 2 and 3; the larger radius and the reflecting dielectric surface, respectively, prevent the transition from a ring to central spot. The loop is also absent in the CVC of case 4 . The CVC of cases 1,2 and 4 (the ones with absorbing dielectric surface) have small humps in range I, although this cannot be seen in the scale of figure 2 .

\section{$3.23 \mathrm{D}$ modes}

Figure 3 displays the CVC of the fundamental mode for condition set 1, points of bifurcation of 3D modes, and an example 3D mode. Each pair of points $a_{i}$ and $b_{i}$ designates from where a $3 \mathrm{D}$ modes branches off from and rejoins the fundamental mode. Mode $a_{i} b_{i}$ possesses period $2 \pi / i$, meaning that $a_{1} b_{1}$ possesses azimuthal period $2 \pi$, mode $a_{2} b_{2}$ possesses azimuthal period $\pi$, and so on. Bifurcation points $b_{1}$ to $b_{4}$ virtually coincide. Points $b_{5}$ and $b_{6}$ are positioned on the section between the turning points. The 3D modes branch off and rejoin the fundamental mode in a palindromic order along current, which conforms to previous modelling of discharges with parallel-plane electrodes.

As an example, the CVC of mode $a_{3} b_{3}$ is shown in figure 3. (The schematic in the figure illustrates the pattern of spots associated with this mode.) The CVC manifests a plateau between $60 \mathrm{~A} \mathrm{~m}^{-2}$ and $300 \mathrm{~A} \mathrm{~m}^{-2}$, which is a manifestation of the normal current density effect. Note that the plateau also is present in the computed mode of the same azimuthal period for a vessel with parallel-plane electrodes and reflecting dielectric surface $[15]$.

CVCs of different modes would be difficult to distinguish in figure 3. A more convenient representation is shown in figure 4: the fundamental and four non-fundamental modes are mapped in the plane $\left(\langle j\rangle, j_{c}\right)$, where $j_{c}$ is current density at a point which is positioned on the upper vertical radius (with respect to the orientation of every schematic) at $r=0.4$ $\mathrm{mm}$, which is the radius of the ring spot in the Townsend discharge. Note that such choice ensures maximum distinction between the modes. Following the fundamental mode from low to high currents, it is seen that $j_{c}$ decreases while the central spot is forming, then it increases as the ring mode forms, thus yielding a limp Z-shape on the bifurcation diagram. Modes $a_{5} b_{5}, a_{6} b_{6}$ possess turning points.

In figure 5 the evolution is shown of spot patterns associated with modes $a_{3} b_{3}, a_{4} b_{4}$, $a_{5} b_{5}, a_{6} b_{6}$ from figure 4 as discharge current is changed. Let us consider first the evolution of the patterns for mode $a_{3} b_{3}$ which is shown in figure $5 \mathrm{a}$ ). The state $303.06 \mathrm{~A} \mathrm{~m}^{-2}$ is positioned in the vicinity of the bifurcation point $b_{3}$, the pattern is of three diffuse elongated spots, slightly deforming into a $3 \mathrm{D}$ structure with the period of $2 \pi / 3$. At $296.06 \mathrm{~A} \mathrm{~m}^{-2}$ the three spots have moved away from each other and become more distinct, intense and bean-shaped. The spots then become circular, and move farther from the center of the cathode as seen in state $36.1 \mathrm{~A} \mathrm{~m}^{-2}$. The spots then once more become bean-shaped, then once again gain a triangular type structure as in state $4.4 \mathrm{~A} \mathrm{~m}^{-2}$. At state $4.4 \mathrm{~A} \mathrm{~m}^{-2}$ a central triangle-shaped 'cold spot' is present. Continuing along the mode with decreasing current, the triangle shaped region becomes less sharp, and the whole pattern becomes more like the ring-shaped distribution present at $a_{3}$.

The evolution of the patterns associated with modes $a_{4} b_{4}, a_{5} b_{5}$, and $a_{6} b_{6}$, is shown in figures $5 \mathrm{~b}-\mathrm{d}$, respectively, follows the same trend as mode $a_{3} b_{3}$ : first the ring is transformed into elongated bean-shaped spots and then circular spots, then they migrate to a different 
radius, and there, from circular spots they turn into bean-shaped spots and then merge into a different ring. No 3D modes with central hot spots were found in the present work, while in previous modelling they were; e.g. [15]. The images in figure 5 can be compared to experimentally observed patterns of spots, figure 2 of [10]. The computed evolution from the abnormal mode into mode $a_{4} b_{4}$, comprising four spots, is in good agreement with the experimentally observed transition between the abnormal mode into a mode comprising four spots.

In figure 2 of [10], it can be seen how the modes appear in the experiment: starting from the abnormal mode and reducing discharge current, a mode comprising four spots appears. As current is further reduced, modes comprising five and six spots appear. Further reducing current from the mode with six spots, the discharge goes back to modes with five, four, three and a ring spot. In the modelling, cf. figure 3, starting from a state in the abnormal mode and following the fundamental mode in the direction of low currents, the bifurcation point $b_{1}$ of mode $a_{1} b_{1}$, comprising one spot, appears first. The next bifurcation point to appear is $b_{2}$ of mode $a_{2} b_{2}$, comprising two spots; and so on until bifurcation point $b_{6}$ of mode $a_{6} b_{6}$, comprising six spots, following the same trend observed in the experiment. On further following the fundamental mode in the direction of low currents, eventually the bifurcation point $a_{5}$ of mode $a_{5} b_{5}$ appears; and so on until $a_{1}$ of mode $a_{1} b_{1}$, again following the same trend as in the experiment.

\subsection{Effect of a reflective dielectric surface}

In figure 6 mode 3 from figure 2 and three non-fundamental modes computed with a dielectric surface that reflects ions and electrons are shown. (In figure $6, j_{c}$ is the current density occurring on the periphery of the cathode at $r=R$ ). Similarly to the case of absorbing dielectric surface, the patterns comprise the axially symmetric ring spot or circular arrangements of 3D spots.

Both the axially symmetric ring spot and 3D spots are centered at the periphery of the cathode in this case, similarly to what happens in the similar modes in the parallelplane electrode configuration with reflecting lateral wall [15]. On the other hand, this is in contrast to what is observed in the experiment and in the modelling with the dielectric surface absorbing the charged particles, where the spots are shifted away from the periphery of the cathode rather than centered at the periphery.

\section{Conclusions}

Self-organized 3D spot modes have been computed for cathode boundary layer discharges. The general form of the computed self-organized patterns is similar to those computed previously in the parallel-plane configuration and to those observed in the experiment in the sense that all of them comprise axially symmetric ring spots or circular arrangements of 3D spots. This is consistent with experimental evidence [4] that similar self-organized patterns appear in both electrode configurations.

Simulations of 3D spot patterns with the dielectric surface fully absorbing the charged particles reveal spots not centered at the periphery of the cathode, but rather located inside the cathode, as they are in the experiment. It has been found that there is a palindromic series of the number of spots with discharge current, which is consistent with 
observations of switching between modes with different patterns in the experiment [10].

\section{Acknowledgments}

The work was supported by FCT - Fundação para a Ciência e a Tecnologia of Portugal through the projects PTDC/FIS-PLA/2708/2012 and Pest-OE/UID/FIS/50010/2013.

\section{References}

[1] K. H. Schoenbach, M. Moselhy, and W. Shi, Plasma Sources Sci. Technol. 13, 177 (2004).

[2] M. Moselhy and K. H. Schoenbach, J. Appl. Phys. 95, 1642 (2004).

[3] N. Takano and K. H. Schoenbach, Plasma Sources Sci. Technol. 15, S109 (2006).

[4] N. Takano and K. H. Schoenbach, in Abstracts of the 2006 IEEE International Con-ference on Plasma Science (IEEE, Traverse City, MI, USA, 2006) p. 247.

[5] B.-J. Lee, H. Rahaman, K. Frank, L. Mares, and D.-L. Biborosch, in Proc. 28th ICPIG (Prague, July 2007), edited by J. Schmidt, M. Šimek, S. Pekárek, and V. Prukner (Institute of Plasma Physics AS CR, ISBN 978-80-87026-01-4, Prague, 2007) pp. 900-902.

[6] W. Zhu, N. Takano, K. H. Schoenbach, D. Guru, J. McLaren, J. Heberlein, R. May, and J. R. Cooper, J. Phys. D: Appl. Phys. 40, 3896 (2007).

[7] B.-J. Lee, D.-L. Biborosch, K. Frank, and L. Mares, J. Optoelectron. Adv. Mater.10, 1972 (2008).

[8] K. H. Schoenbach and W. Zhu, IEEE J. Quantum. Electron. 48, 768 (2012).

[9] W. Zhu, P. Niraula, P. G. C. Almeida, M. S. Benilov, and D. F. N. Santos, Plasma Sources Sci. Technol. 23, 054012 (2014).

[10] W. Zhu and P. Niraula, Plasma Sources Sci. Technol. 23, 054011 (2014).

[11] P. G. C. Almeida, M. S. Benilov, M. D. Cunha, and M. J. Faria, J. Phys. D: Appl. Phys. 42, 194010 (21pp) (2009).

[12] P. G. C. Almeida, M. S. Benilov, and M. J. Faria, Plasma Sources Sci. Technol. 19, 025019 (13pp) (2010).

[13] P. G. C. Almeida, M. S. Benilov, and M. J. Faria, IEEE Trans. Plasma Sci. 39, 2190 (2011).

[14] P. G. C. Almeida and M. S. Benilov, Phys. Plasmas 20, 101613 (2013).

[15] P. G. C. Almeida, M. S. Benilov, and D. F. N. Santos, ArXiv e-prints (2014), arXiv:1406.4394 .

[16] M. S. Benilov, Plasma Sources Sci. Technol. 23, 054019 (2014).

[17] Yu. P. Raizer, Gas Discharge Physics (Springer, Berlin, 1991).

\section{Figures}

Figure 1: Configuration of a cathode boundary layer vessel. AG is an axis of symmetry.

Figure 2: Fundamental mode, Xenon, 30 Torr, CBLD configuration of figure 1. 1: $\mathrm{h}=0.5 \mathrm{~mm}, \mathrm{~h}_{a}=0.1 \mathrm{~mm}, \mathrm{R}=0.5 \mathrm{~mm}$, absorbing lateral wall. $2: \mathrm{h}=0.5 \mathrm{~mm}, \mathrm{~h}_{a}=0.1 \mathrm{~mm}$, 
$\mathrm{R}=1.5 \mathrm{~mm}$, absorbing lateral wall. $3: \mathrm{h}=0.5 \mathrm{~mm}, \mathrm{~h}_{a}=0.1 \mathrm{~mm}, \mathrm{R}=0.5 \mathrm{~mm}$, reflecting lateral wall. $4: \mathrm{h}=0.25 \mathrm{~mm}, \mathrm{~h}_{a}=0.25 \mathrm{~mm}, \mathrm{R}=0.375 \mathrm{~mm}$, absorbing lateral wall.

Figure 3: CVC, xenon, 30 Torr, $\mathrm{h}=0.5 \mathrm{~mm}, \mathrm{~h}_{a}=0.1 \mathrm{~mm}, \mathrm{R}=0.5 \mathrm{~mm}$, absorbing lateral wall. Solid: Fundamental mode (mode 1 of figure 2 ). Dashed: mode $a_{3} b_{3}$. Circles: points of bifurcation.

Figure 4: Bifurcation diagram, xenon, 30 Torr, $\mathrm{h}=0.5 \mathrm{~mm}, \mathrm{~h}_{a}=0.1 \mathrm{~mm}, \mathrm{R}=0.5 \mathrm{~mm}$, absorbing lateral wall. Solid: Fundamental mode (mode 1 of figure 2). Dashed: modes $a_{3} b_{3}, a_{4} b_{4}, a_{5} b_{5}$. Dotted: mode $a_{6} b_{6}$. Circles: points of bifurcation.

Figure 5: Evolution of patterns associated with 3D modes of figure 4. a) mode $a_{3} b_{3}$ b) mode $a_{4} b_{4}$ c) mode $a_{5} b_{5}$ d) mode $a_{6} b_{6}$.

Figure 6: Bifurcation diagram, xenon, 30 Torr, $\mathrm{h}=0.5 \mathrm{~mm}, \mathrm{~h}_{a}=0.1 \mathrm{~mm}, \mathrm{R}=0.5 \mathrm{~mm}$, reflecting lateral wall. Solid: fundamental mode (mode 3 from figure 2). Dashed: modes $a_{3} b_{3}, a_{6} b_{6}, a_{10} b_{10}$. Circles: points of bifurcation. 
This figure "figure1.png" is available in "png" format from: http://arxiv.org/ps/1510.07530v2 
This figure "figure2.png" is available in "png" format from: http://arxiv.org/ps/1510.07530v2 
This figure "figure3.png" is available in "png" format from: http://arxiv.org/ps/1510.07530v2 
This figure "figure4.png" is available in "png" format from: http://arxiv.org/ps/1510.07530v2 
This figure "Figure5.png" is available in "png" format from: http://arxiv.org/ps/1510.07530v2 
This figure "Figure6.png" is available in "png" format from: http://arxiv.org/ps/1510.07530v2 